\title{
Isolated optic neuritis with a concurrent abnormal trigeminal nucleus on imaging: case report of a rare complication of herpes zoster ophthalmicus
}

Kavin Vanikieti', Anuchit Poonyathalang ${ }^{1}$, Panitha Jindahra², Piyaphon Cheecharoen ${ }^{3}$, Patchalin Patputtipong ${ }^{3}$ and Tanyatuth Padungkiatsagul ${ }^{1 *}$ (i)

\begin{abstract}
Background: Herpes zoster ophthalmicus (HZO) is an inflammation related to reactivation of the latent varicella zoster virus (VZV), involving the ophthalmic branch of the trigeminal nerve. Optic neuritis (ON), a rare ocular complication following $\mathrm{HZO}$, has been reported in 1.9\% of HZO-affected eyes. Most previous cases occurred simultaneously with other ocular complications, especially orbital apex syndrome. Moreover, detailed magnetic resonance imaging (MRI) with diffusion weighted imaging of the optic nerve and trigeminal nucleus in HZOrelated ON has been rarely reported. We report a case of postherpetic isolated ON with a concurrent abnormal trigeminal nucleus on imaging.
\end{abstract}

Case presentation: A healthy 58-year-old female presented with sudden painful visual loss in her right eye for 2 days. Four weeks before the presentation, her right eye was diagnosed with $\mathrm{HZO}$, and she received intravenous acyclovir for 10 days. Ophthalmic examination revealed a visual acuity of light perception and 20/20 in the right and left eyes, respectively. A relative afferent pupillary defect was present in the right eye. Neurological examination was significant for hypoesthesia in the area of the HZO. A clinical diagnosis of HZOrelated right retrobulbar ON was made, and other causes of atypical ON were excluded. MRI showed enhancement and restricted diffusion of the right-sided optic nerve with linear hyperintense T2 of the right-sided spinal trigeminal nucleus and tract (STNT) along the brainstem. She received 14 days of intravenous acyclovir and 5 days of methylprednisolone. Both were switched to an oral route for 2 months. After the completion of treatment, the visual acuity was counting fingers and 20/20 in the right eye and left eye, respectively. Stable brainstem STNT abnormalities and resolution of ON were found radiologically.

Conclusions: Isolated $\mathrm{ON}$ is a rare ocular complication following $\mathrm{HZO}$. An abnormal high signal of STNT on a T2 weighted image may be present, which may be a clue for VZV-associated complications, such as HZOrelated ON, especially in cases lacking an obvious history of $\mathrm{HZO}$ or other concomitant ocular complications. Prompt treatment with both acyclovir and corticosteroids should be started. Restricted diffusion of the optic nerve may be a predictor for poor visual recovery.

Keywords: Herpes zoster ophthalmicus, Optic neuritis, Magnetic resonance imaging, Diffusion weighted imaging, Trigeminal nucleus

\footnotetext{
* Correspondence: Blu_c16@hotmail.com

'Department of Ophthalmology, Faculty of Medicine Ramathibodi Hospital,

Mahidol University, 270 Rama VI Road, Bangkok 10400, Thailand

Full list of author information is available at the end of the article
}

(c) The Author(s). 2018 Open Access This article is distributed under the terms of the Creative Commons Attribution 4.0 International License (http://creativecommons.org/licenses/by/4.0/), which permits unrestricted use, distribution, and reproduction in any medium, provided you give appropriate credit to the original author(s) and the source, provide a link to the Creative Commons license, and indicate if changes were made. The Creative Commons Public Domain Dedication waiver (http://creativecommons.org/publicdomain/zero/1.0/) applies to the data made available in this article, unless otherwise stated. 


\section{Background}

Herpes zoster ophthalmicus (HZO) is an inflammation related to reactivation of the latent varicella zoster virus (VZV) involving the ophthalmic branch of the trigeminal nerve [1]. Ocular complications are found in $50 \%$ of $\mathrm{HZO}$ patients, following the onset of a rash in 1-4 weeks [2].

Any ocular structures can be affected, although anterior uveitis and keratitis are the most common ocular complications [3]. Optic neuritis (ON), a rare ocular complication following $\mathrm{HZO}$, has been reported in 1.9\% of HZO-affected eyes [3]. However, HZO-related ON in many previous reports was not isolated. Most of the previous cases occurred simultaneously with other ocular complications, especially orbital apex syndrome $[4,5]$. Moreover, detailed magnetic resonance imaging (MRI) with diffusion weighted imaging (DWI) of the optic nerve and trigeminal nucleus in HZO-related $\mathrm{ON}$ has been rarely documented. Herein, we report a case of postherpetic isolated $\mathrm{ON}$ with a concurrent abnormal trigeminal nucleus on imaging.

\section{Case presentation}

A previously healthy 58 -year-old female presented to our clinic with a sudden painful visual loss in her right eye for 2 days. Ocular movement significantly aggravated her pain. Four weeks before the presentation, she developed a group of vesicles on the erythematous base over the right ophthalmic branch of the trigeminal nerve including the tip of her nose, which was diagnosed as HZO. At that time, she was treated with intravenous acyclovir $(30 \mathrm{mg} / \mathrm{kg} /$ day) for 10 days. The group of vesicles soon disappeared and turned to hyperpigmented macules and patches (Fig. 1).

At our clinic, an ophthalmic examination revealed best-corrected visual acuity of light perception in the right eye, compared with 20/20 in the left eye. A relative afferent pupillary defect (RAPD) was present in the right eye. Intraocular pressures were $12 \mathrm{mmHg}$ in both eyes. Ocular motility, anterior segment, and a fundus examination were unremarkable bilaterally. Neither proptosis nor ptosis was observed. The neurological examination was significant for hypoesthesia in the area supplied by the right ophthalmic branch of the trigeminal nerve. A clinical diagnosis of HZO-related right retrobulbar ON was made. To exclude other possible causes of atypical ON, a blood test including a complete blood count (CBC), erythrocyte sedimentation rate (ESR), c-reactive protein (CRP), Venereal Disease Research Laboratory (VDRL), Treponema pallidum hemagglutination (TPHA), antinuclear antibody (ANA), and aquaporin 4-antibody were performed, which all showed normal results. MRI of the brain and orbit showed enhancement and restricted diffusion of a right-sided intraorbital, intracanalicular, and prechiasmatic optic nerve (Fig. 2). Notably, linear hyperintense T2 lesions in

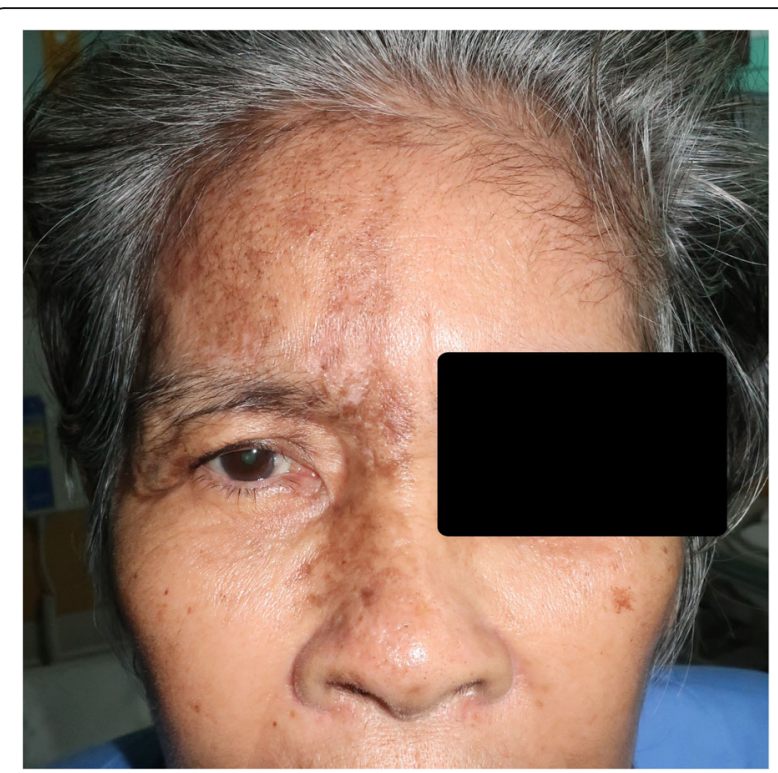

Fig. 1 External appearance. Hyperpigmented macules and patches over the right ophthalmic branch of the trigeminal nerve, including the tip of the nose

vertical orientation extending from the right dorsolateral pons down to the medulla without any enhancement or restricted diffusion were also found (Fig. 3). These vertical lesions represented the anatomical location of the spinal trigeminal nucleus and tract (STNT) along the brainstem. Lumbar puncture showed mild lymphocytic pleocytosis (22 cells, 98\% lymphocytes) with normal protein and a negative polymerase chain reaction (PCR) for VZV.

Treatment was started with intravenous acyclovir (30 $\mathrm{mg} / \mathrm{kg} /$ day) along with $1 \mathrm{~g} /$ day of intravenous methylprednisolone. Intravenous acyclovir was continued for 14 days, then reduced to $800 \mathrm{mg}$ oral acyclovir daily. Acyclovir was discontinued in the third month. Oral prednisolone $(1 \mathrm{mg} / \mathrm{kg} /$ day $)$ was started after 5 days of intravenous methylprednisolone, then gradually tapered and discontinued in the third month. After the completion of the 2 month treatment, the best-corrected visual acuity was counting fingers and 20/20 in the right and left eyes, respectively. An ophthalmic examination detected a right optic disc atrophy with normal physiological cupping. MRI of the brain and orbit showed stable brainstem STNT abnormalities and resolution of the ON.

\section{Discussion and conclusions}

$\mathrm{ON}$ is an unusual ocular complication secondary to HZO that may present either in papillitis or retrobulbar form and occur weeks to months after the onset of the rash [6-12]. The degree of visual loss varies, ranging from mild to severe visual impairment [6-12]. Adults, especially the elderly, are more affected; however, HZO-related ON in children has also been reported $[9,13]$. 


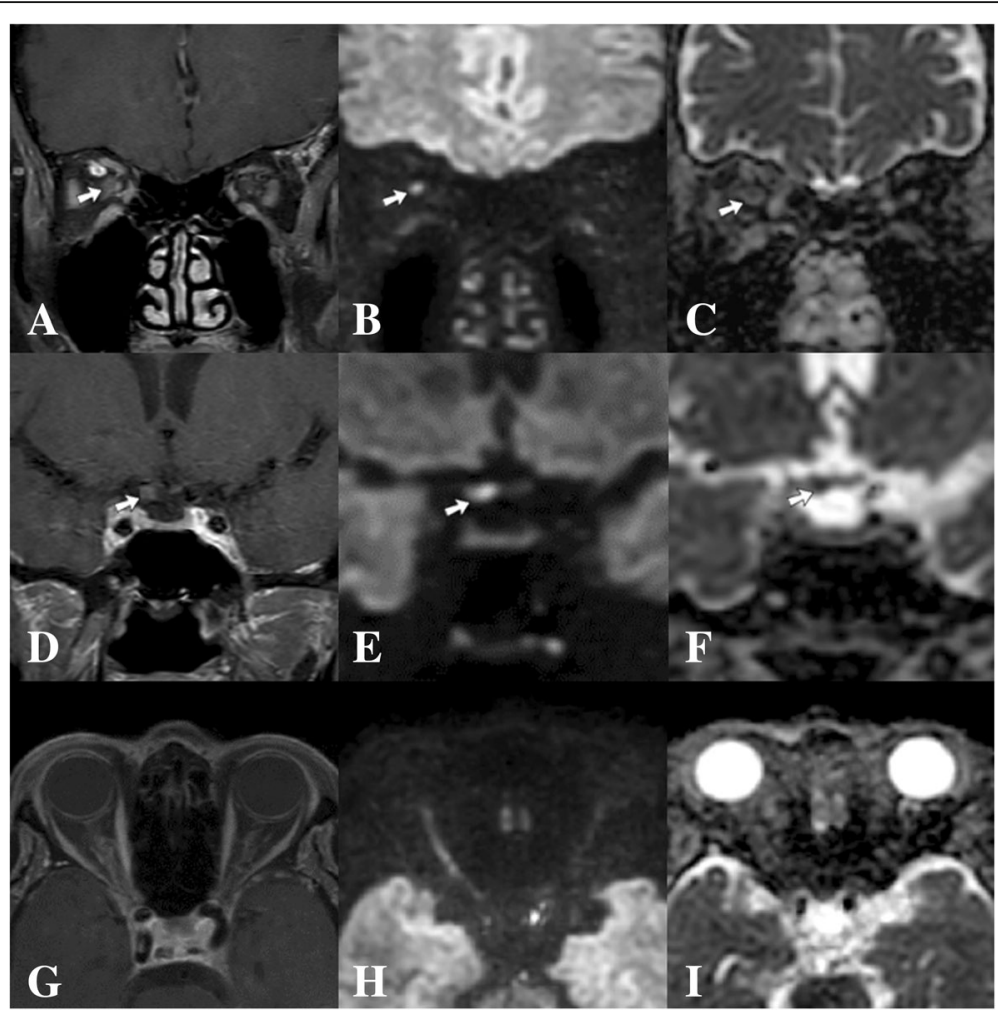

Fig. 2 Magnetic resonance imaging of the orbit with diffusion weighted imaging (DWI) and an apparent diffusion coefficient (ADC). Coronal T1 weighted with a gadolinium injection (a) shows enhancement of the right intraorbital optic nerve (arrow) along with restricted diffusion (arrow) on DWI (b), which was confirmed by the low signal (arrow) on the ADC map (c). Coronal T1 weighted with a gadolinium injection (d) shows enhancement of the right prechiasmatic optic nerve (arrow) along with restricted diffusion (arrow) on DWI (e), which was confirmed by the low signal (arrow) on the ADC map (f). Axial T1 weighted with a gadolinium injection (g) shows enhancement of the right optic nerve along with restricted diffusion on DWI (h), which was confirmed by the low signal on the ADC map (i)

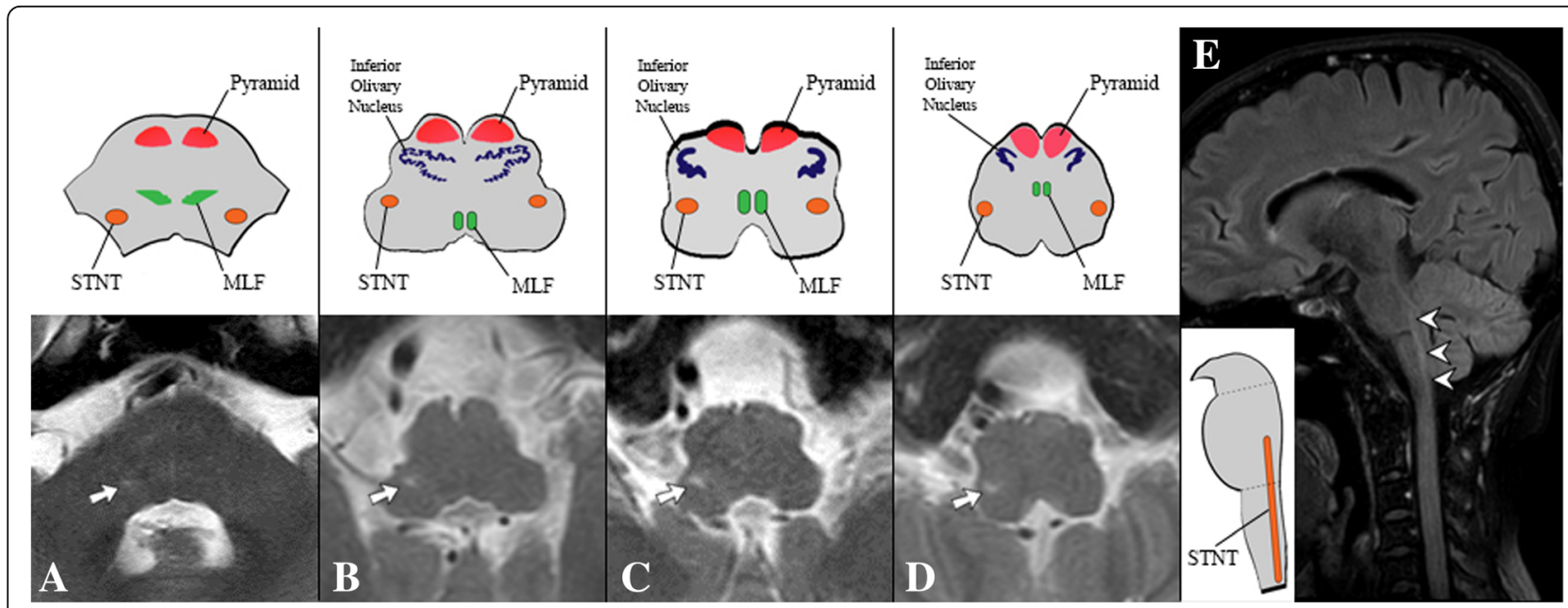

Fig. 3 Magnetic resonance imaging of the brain. Axial T2 weighted shows hyperintense T2 lesions (arrows) extending from right dorsolateral pons to the medulla (a-d). Sagittal fluid attenuation inversion recovery (FLAIR) shows linear, vertical-oriented hyperintense lesions (arrowheads) (e). These lesions represent the anatomical location of the spinal trigeminal nucleus and tract (STNT) along the brainstem. MLF, medial longitudinal fasciculus 
HZO-related ON in many previous reports was found simultaneously with other ocular complications, such as anterior uveitis, keratitis, and secondary glaucoma. Moreover, it may occur as part of the orbital apex syndrome $[4,5]$. Our case uniquely demonstrated an isolated $\mathrm{HZO}$-related $\mathrm{ON}$ without any concomitant ocular complications, which has been rarely reported.

Despite the absence of VZV in the cerebrospinal fluid (CSF) using the PCR, a diagnosis of HZO-related ON was made based on the following:

(1) The concordance in laterality between the HZO and $\mathrm{ON}$.

(2) Nasociliary nerve involvement of HZO based on the presence of hyperpigmented macules and patches over the tip of the nose, which was highly associated with the development of ocular complications [2].

(3) A temporal relationship between the $\mathrm{HZO}$ and $\mathrm{ON}$.

(4) A high signal of STNT on the T2 weighted MRI.

(5) The exclusion of other possible causes of atypical $\mathrm{ON}$.

The precise mechanism of ON following HZO is controversial. Viral replication in the ophthalmic branch of the trigeminal nerve, located in the cavernous sinus, spreads through the superior orbital fissure to the orbit, where it may cause direct injury to the optic nerve [14]. Extensive inflammation involving posterior ciliary arteries and nerves based on histopathology of HZO-affected eyes, which may lead to ocular ischemia and subsequent optic nerve damage, is another possible mechanism [15]. Parainfectious $\mathrm{ON}$, as a consequence of a self-immune response triggered by the VZV antigen, has also been postulated [16]. These proposed mechanisms are usually difficult to differentiate from each other.

MRI findings of the optic nerve in HZO-related ON have been rarely described. Peripheral enhancement of the intraorbital optic nerve sheath has been commonly reported $[4,5,12]$. In our case, not only the optic nerve sheath, but also the axial portion of the optic nerve itself was affected. Moreover, the enhancement extended to the intracanalicular and prechiasmatic optic nerve. These results are consistent with the results of Wang et al., suggesting that these findings explained the severe visual impairment of $\mathrm{ON}$ and poor visual outcome in our case [12]. To our knowledge, DWI findings of the optic nerve have never been reported in HZO-related ON. However, restriction of the optic nerve on DWI, which is consistent with infarction attributed to compression or inflammation of the vessels serving the optic nerve, are rarely present in ON $[17,18]$. Our case demonstrated a very long restricted diffusion of the optic nerve, including the entire intraorbital segment extending to the prechiasmatic segment. This may be a predictor for poor visual recovery in our case.

We found an unusually high signal of STNT on the T2 weighted MRI. This is thought be a result of VZV migration from the gasserian ganglion to the STNT along the brainstem [5, 19-21]. Douglas et al. reported contiguous hyperintense T2 lesions with restricted diffusion on DWI along the brainstem, which corresponded to the anatomical location of STNT in a VZV encephalitis patient [21]. Two months following complete anti-viral treatment, these lesions improved [21]. In our case, STNT failed to show any restriction on DWI. However, the restriction of the right-sided optic nerve observed in our case might be compatible with the restricted diffusion of the STNT reported by Douglas et al. [21].

Because the proposed mechanism of HZO-related ON is poorly understood and more than one mechanism can occur, it is reasonable to start the treatment with a combination of acyclovir and corticosteroids. In many previous reports, the duration of treatment varied, ranging from 10 days to 2 months [6-12]. We decided to continue the treatment up to 2 months because of the severe visual impairment of $\mathrm{ON}$.

Visual recovery in ON following HZO is typically excellent [6-12]. Nevertheless, severe visual outcomes have also been reported [3]. Concomitant ocular complications, such as retinitis and secondary glaucoma may contribute to a poor visual prognosis. Based on the MRI with DWI of the optic nerve, we hypothesized that poor visual recovery in our case was due to the following:

(1) Both axial and optic nerve sheath portions were affected.

(2) A very long enhancement with restricted diffusion of the optic nerve occurred.

In summary, we report a case of isolated $\mathrm{ON}$ following $\mathrm{HZO}$ along with restricted diffusion of the optic nerve on imaging. In addition, an abnormal high signal of STNT on the T2 weighted image was found. This may be a clue of $\mathrm{VZV}$-associated complications, such as $\mathrm{HZO}$-related ON, especially in cases lacking an obvious history of $\mathrm{HZO}$ or other concomitant ocular complications. Prompt treatment with both acyclovir and corticosteroids should be started. Restricted diffusion of the optic nerve may therefore be a predictor for poor visual recovery.

\section{Abbreviations}

ANA: Antinuclear antibody; CBC: Complete blood count; CRP: C-reactive protein; CSF: Cerebrospinal fluid; DWI: Diffusion weighted imaging; ESR: Erythrocyte sedimentation rate; HZO: Herpes zoster ophthalmicus; MLF: Medial longitudinal fasciculus; MRI: Magnetic resonance imaging; ON: Optic neuritis; PCR: Polymerase chain reaction; RAPD: Relative afferent pupillary defect; STNT: Spinal trigeminal nucleus and tract; TPHA: Treponema pallidum hemagglutination; VDRL: Venereal Disease Research Laboratory; VZV: Varicella zoster virus 


\section{Acknowledgements}

We thank Larry Takemoto, PhD, from Edanz Group (www.edanzediting.com/ac) for editing a draft of this manuscript.

\section{Funding}

None.

\section{Availability of data and materials}

Data for this case report were collected by chart review of the patient's electronic medical record, which was not publicly available because of privacy considerations

\section{Authors' contributions}

Substantial contributions to the conception (KV and TP) or design (KV and TP) of the work, or the acquisition (PC and TP), analysis (KV, AP, PJ, PC, PP, and TP) or interpretation (KV, PC, PP, and TP) of data. Drafting the work (KV) or revising it critically (KV). Final approval for submission (KV, AP, PJ, PC, PP, and TP).

\section{Ethics approval and consent to participate}

This study was approved by the Institutional Review Board of Faculty of Medicine Ramathibodi Hospital and the participant gave written informed consent prior to entering the study.

\section{Consent for publication}

Written informed consent was obtained from the patient for publication of this case report and any accompanying images. A copy of the consent form is available for review by the Editor of this journal.

\section{Competing interests}

The author declare that they have no competing interests.

\section{Publisher's Note}

Springer Nature remains neutral with regard to jurisdictional claims in published maps and institutional affiliations.

\section{Author details}

'Department of Ophthalmology, Faculty of Medicine Ramathibodi Hospital, Mahidol University, 270 Rama VI Road, Bangkok 10400, Thailand. 2Department of Medicine, Faculty of Medicine Ramathibodi Hospital, Mahidol University, 270 Rama VI Road, Bangkok 10400, Thailand. ${ }^{3}$ Department of Radiology, Faculty of Medicine Ramathibodi Hospital, Mahidol University, 270 Rama VI Road, Bangkok 10400, Thailand.

Received: 10 January 2018 Accepted: 28 September 2018 Published online: 04 October 2018

\section{References}

1. Weller TH. Varicella and herpes zoster. Changing concepts of the natural history, control, and importance of a not-so-benign virus. N Engl J Med. 1983;309:1434-40.

2. Harding SP, Lipton JR, Wells JC. Natural history of herpes zoster ophthalmicus: predictors of postherpetic neuralgia and ocular involvement. Br J Ophthalmol. 1987;71:353-8.

3. Kahloun R, Attia S, Jelliti B, Attia AZ, Khochtali S, Yahia SB, Zaouali S, Khairallah M. Ocular involvement and visual outcome of herpes zoster ophthalmicus: review of 45 patients from Tunisia, North Africa. J Ophthalmic Inflamm Infect. 2014;4:25,

4. Lee CY, Tsai HC, Lee SS, Chen YS. Orbital apex syndrome: an unusual complication of herpes zoster ophthalmicus. BMC Infect Dis. 2015;15:33.

5. Paraskevas GP, Anagnostou E, Vassilopoulou S, Spengos K. Painful ophthalmoplegia with simultaneous orbital myositis, optic and oculomotor nerve inflammation and trigeminal nucleus involvement in a patient with herpes zoster ophthalmicus. BMJ Case Rep. 2012. https://doi.org/10.1136/ bcr-2012-007063.

6. Deane JS, Bibby K. Bilateral optic neuritis following herpes zoster ophthalmicus. Arch Ophthalmol. 1995;113:972-3.

7. de Mello Vitor B, Foureaux EC, Porto FB. Herpes zoster optic neuritis. Int Ophthalmol. 2011;31:233-6.

8. Yalcinbayir O, Gelisken O, Yilmaz E. Unilateral optic neuritis in a case of herpes zoster ophthalmicus. Neuroophthalmology. 2009;33:339-42.
9. Hong SM, Yang YS. A case of optic neuritis complicating herpes zoster ophthalmicus in a child. Korean J Ophthalmol. 2010;24:126-30.

10. Freitas-Neto CA, Cerón O, Pacheco KD, Pereira VO, Ávila MP, Foster CS. Optic neuritis complicating herpes zoster ophthalmicus in an immunocompetent patient. Rev Bras Oftalmol. 2014;73:386-8.

11. Singh P, Karmacharya S, Rizyal A, Rijal AP. Herpes zoster ophthalmicus with retrobulbar neuritis. Nepal J Ophthalmol. 2016;8:78-81.

12. Wang AG, Liu JH, Hsu WM, Lee AF, Yen MY. Optic neuritis in herpes zoster ophthalmicus. Jpn J Ophthalmol. 2000;44:550-4.

13. Monroe LD. Optic neuritis in a child with herpes zoster. Ann Ophthalmol. 1979;11:405.

14. Gündüz K, Özdemir Ö. Bilateral retrobulbar neuritis following unilateral herpes zoster ophthalmicus. Ophthalmologica. 1994;208:61-4.

15. Naumann G, Donald J, Gass M, Font RL. Histopathology of herpes zoster ophthalmicus. Am J Ophthalmol. 1968:65:533-41.

16. Pless ML, Malik SI. Relapsing-remitting, corticosteroid-sensitive, varicella zoster virus optic neuritis. Pediatr Neurol. 2003;29:422-4.

17. Spierer O, Ben Sira L, Leibovitch I, Kesler A. MRI demonstrates restricted diffusion in distal optic nerve in atypical optic neuritis. J Neuroophthalmol. 2010;30:31-3.

18. Bender B, Heine C, Danz S, Bischof F, Reimann K, Bender M, Nägele T, Ernemann $U$, Korn A. Diffusion restriction of the optic nerve in patients with acute visual deficit. J Magn Reson Imaging. 2014;40:334-40.

19. Haanpää M, Dastidar P, Weinberg A, Levin M, Miettinen A, Lapinlampi A, Laippala P, Nurmikko T. CSF and MRI findings in patients with acute herpes zoster. Neurology. 1998;51:1405-11.

20. Siritho S, Pumpradit W, Suriyajakryuththana W, Pongpirul K. Severe headache with eye involvement from herpes zoster ophthalmicus, trigeminal tract, and brainstem nuclei. Case Rep Radiol. 2015. https://doi.org/10.1155/2015/402015.

21. Douglas JE, Buch VP, Mamourian AC. Varicella zoster-induced magnetic resonance imaging abnormalities of the trigeminal nucleus. J Neurol Sci. 2015;359:57-8.

\section{Ready to submit your research? Choose BMC and benefit from:}

- fast, convenient online submission

- thorough peer review by experienced researchers in your field

- rapid publication on acceptance

- support for research data, including large and complex data types

- gold Open Access which fosters wider collaboration and increased citations

- maximum visibility for your research: over $100 \mathrm{M}$ website views per year

At BMC, research is always in progress.

Learn more biomedcentral.com/submission 